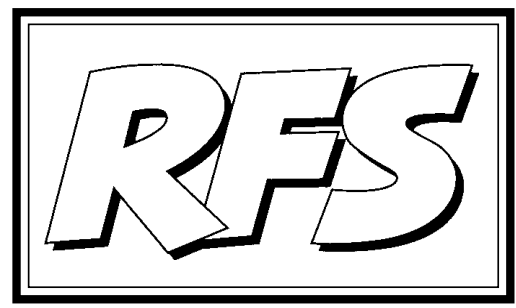

Revista de Fomento Social, 53 (1998), 47-68

\title{
La política agraria en el proceso de integración europea
}

El objetivo de este artículo es analizar el proceso de elaboración, aplicación y posterior reforma de la política agraria común (PAC) de la Unión Europea (UE), estudiando los factores sociales, políticos y económicos que han incidido en dicho proceso. Este análisis responde a una reflexión más general sobre las relaciones entre Estado y sociedad civil en contextos democráticos ya consolidados, como lo era el de los seis países que constituyeron la entonces denominada Comunidad Económica Europea (CEE) allá por finales de los años cincuenta -Francia, República Federal de Alemania, Italia, Bélgica, Países Bajos y Luxemburgo-, y como lo es el de los quince que componen la actual UE. En esos contextos, la formulación y puesta en marcha de políticas públicas suele tener efectos de reestructuración social al favorecer la emergencia de nuevos actores colectivos y la constitución de marcos institucionales para facilitar su participación en los procesos de toma de decisiones.

$(*)$

(*) IESA-CSIC de Andalucía. Córdoba. España. Este artículo es la versión actualizada de una conferencia pronunciada por el autor en septiembre de 1996 en la Universidad de Santa María (Río Grande del Sur. Brasil). 
El caso de la agricultura europea y la PAC puede considerarse un caso ilustrativo para reflexionar sobre el problema de las relaciones Estado/sociedad civil en democracias avanzadas, sobre todo en etapas de transición hacia nuevos modelos de desarrollo económico y social, etapas en las que se pasan de viejas a nuevas políticas públicas, y en las que coexisten viejos y nuevos actores colectivos que pugnan por erigirse en representantes del sector afectado por dichas políticas.

El desarrollo de este artículo se hará exponiendo, en primer lugar, los elementos fundamentales del contexto en el que emerge el proceso de construcción europea y la formulación de una política agraria común. En segundo lugar, se analizarán los principios orientadores de la PAC, así como los objetivos perseguidos y los instrumentos puestos en marcha para alcanzarlos. En tercer lugar, se analizará el actual proceso de crisis y reforma de dicha política -una crisis debida al éxito de la mayor parte de sus iniciales planteamientos y no al fracaso de los mismos-, así como la emergencia de nuevas políticas y nuevos actores sociales.

\section{El contexto de la construcción europea}

La Europa de final de los años cincuenta era muy diferente a la de ahora. Desde el punto de vista económico, era una sociedad que se recuperaba de los efectos devastadores del último conflicto bélico sobre su estructura productiva, una sociedad que aún no había alcanzado la autosuficiencia alimentaria y que iniciaba un fuerte proceso de crecimiento y desarrollo en nuevos sectores de actividad.

Desde el punto de vista político, existía la voluntad entre las élites políticas de crear en Europa occidental las condiciones para garantizar un futuro de paz y estabilidad que evitara los constantes enfrentamientos entre países -sobre todo, entre Francia y Alemania-que habían caracterizado la reciente historia europea -las dos últimas guerras mundiales eran vistas como dos guerras civiles en suelo europeo. Para ello, se planteó la conveniencia de crear un espacio político y económico supranacional, es decir, una red de interdependencias entre los distintos Estados como vía de neutralización y mediación de los conflictos que pudieran originarse.

Desde el punto de vista ideológico, el discurso dominante a mediados de los 
años cincuenta era el de la intervención estatal en la economía para facilitar el crecimiento económico, es decir, un discurso keynesiano favorable a la potenciación de las políticas públicas como forma más adecuada para la construcción de un Estado del bienestar en Europa.

En definitiva, productividad, crecimiento económico, estabilidad política, espíritu de consenso e intervención estatal, eran los elementos característicos del contexto en que comenzaría el proceso de construcción europea. El Tratado de Roma, firmado en marzo de 1957, creará la Comunidad Económica Europea (CEE) entre los seis países antes mencionados, como primer paso para la construcción de una unión política que debía estar permanente abierta a la incorporación futura del resto de países europeos con regímenes democráticos.

Como prueba de esa ya inicial vocación de unión política, el Tratado de Roma definía un sistema institucional basado en el modelo de las democracias parlamentarias -con un órgano ejecutivo (Comisión Europea), otro legislativo (repartido entre los Consejos de Ministros y el Parlamento) y otro judicial (Tribunal de Justicia)-, así como un nuevo sistema normativo -constituido por reglamentos, directivas, recomendaciones y decisiones- para la formulación y desarrollo de las distintas políticas comunes.

Mientras que los Consejos de Ministros -uno por cada área políticarepresentaban los intereses de los Estados miembros, al estar constituidos por los correspondientes ministros de los distintos gobiernos nacionales, la Comisión se erigía en el representante de los intereses generales de la CEE, al actuar de forma independiente de dichos gobiernos y teniendo a su servicio una extensa red de funcionarios seleccionados expresamente para desarrollar las tareas técnicoadministrativas encomendadas. El Parlamento, que inicialmente estaba constituido por diputados de los distintos parlamentos nacionales y que gozaba de muy escasas competencias legislativas, excepto la de aprobar el presupuesto de la CEE, pasaría más tarde a estar formado por diputados elegidos directamente por sufragio universal en elecciones europeas, y a aumentar su importancia política en el proceso de toma de decisiones (1). Para marcar las orientaciones del

(1) Cada cuatro años se celebran en todos los países de la UE elecciones para elegir a los diputados del Parlamento europeo. Inicialmente, hasta la extensión del sufragio universal, el Parlamento europeo estaba constituido por diputados de los distintos parlamentos nacionales. 
proceso de construcción europea, los jefes de Estado y de Gobierno comenzaron a reunirse cada seis meses en una ciudad europea, en las llamadas «cumbres europeas», que más tarde acabarían institucionalizándose en la forma de un nuevo órgano político, el actual Consejo Europeo.

Un aspecto importante de ese nuevo sistema institucional sería la creación de órganos consultivos para canalizar la representación de los distintos grupos socioprofesionales, siguiendo la tradición de concertación neocorporativa ya desarrollada en algunos países centroeuropeos. Así, se creó el Comité Económico y Social, como órgano consultivo de carácter general, y los «comités consultivos agrícolas», para desarrollar las prácticas de concertación de tipo sectorial en el ámbito de la política agraria común.

Para adentrarse en la complejidad que supone alcanzar acuerdos entre países y grupos de intereses tan diversos como los que constituían entonces, y constituyen ahora con mayor complejidad si cabe (2), el espacio económico y político europeo, hay que resaltar que todo ese aparato institucional ha venido funcionando desde sus inicios sobre la base de la búsqueda del consenso, característica ésta que ha marcado el proceso de construcción europea, sobre todo a raíz del llamado «espíritu de Luxemburgo» (3). Con esta búsqueda de consenso, la CEE (ComunidadEconómica Europea), primero, la CE(Comunidad Europea), después, y ahora la UE (Unión Europea) han procurado que el avance de la construcción europea se haga sin que ningún país, por pequeño que sea, se vea excluido de las decisiones adoptadas por los órganos políticos comunitarios.

(2) La CEE pasó primero de seis a nueve miembros, con la incorporación de Reino Unido, Dinamarca e Irlanda; más tarde se incorporó Grecia; en 1986 se incorporó España y Portugal, y ahora es una Unión Europea de quince miembros, tras la reciente incorporación de Suecia, Austria y Finlandia. La prevista incorporación de los antiguos países del Este europeo, como Polonia, Hungría y República Checa, la ex-república yugoslava de Eslovenia y Chipre, aumenta la complejidad del espacio político y económico europeo.

(3) En la cumbre de jefes de Estado y de Gobierno celebrada en Luxemburgo en 1967, se acordó, ante la crisis planteada por Francia, que amenazó incluso con salirse de la CEE, la adopción de acuerdos por unanimidad y la posibilidad de ejercer el derecho de veto por parte de algún país que se sintiera marginado del proceso de toma de decisiones. Este acuerdo era de naturaleza política al no estar recogido en el Tratado de Roma, un tratado en el que se contempla que las decisiones se tomen por mayoría, si bien con voto ponderado según los países y de acuerdo al tamaño de su población. 


\section{Principios e instrumentos de la política agraria común}

El Tratado de Roma establecía ya en su artículo 39 el mandato de constituir de forma inmediata una política agraria común, lo que da idea de la importancia que se le daba al hecho de poner en común una política pública como base para que el proceso de construcción europea no se quedara estancado en mera retórica o en simple declaración de intenciones. De acuerdo con el Tratado de Roma, la política agraria común (PAC) debía lograr los objetivos de aumentar la productividad agraria, estabilizar los mercados, equiparar las rentas de los agricultores a las de los demás sectores socioprofesionales, alcanzar la autosuficiencia alimentaria y suministrar alimentos a los consumidores a precios razonables. Para ello, el propio Tratado establecía ya como principios básicos de la futura PAC, los de unidad de mercado -libre circulación de productos y creación de un sistema de precios único-, solidaridad financiera-financiación del coste de dicha política con cargo al presupuesto comunitario y no a los presupuestos nacionales- y preferencia comunitaria-favorecer el consumo de los productos internos frente a la competencia exterior.

La Cumbre de Stressa -que reunió en julio de 1958, es decir, un año después de la firma del Tratado de Roma, a los jefes de Estado y de Gobierno de los seis países miembros de la CEE- aprobaría el conjunto de instrumentos fundamentales de la PAC, iniciándose así el proceso de formulación y puesta en marcha de una política común para la agricultura europea. Entre tales instrumentos destacaban los siguientes. En primer lugar, un sistema de precios de garantía para mantener en un nivel apropiado la renta de los agricultores y evitar crisis cíclicas, con lo que se pretendía alcanzar tres de los objetivos antes mencionados, a saber: equiparar las rentas de los agricultores con las de otros sectores, estabilizar los mercados y ofrecer productos a los consumidores a precios razonables. Los organismos públicos de intervención debían comprar la producción a los agricultores cuando el precio de mercado se colocara por debajo de un precio mínimo, y ordenar importaciones cuando se elevara por encima de un precio máximo. La puesta en práctica de ese instrumento se haría en cada sector productivo a través de las llamadas OCM (Organizaciones Comunes de Mercados).

En segundolugar, un sistema de medidas de protección respecto a la competencia de los productos agrícolas procedentes del exterior -a través de los llamados prélévements-, para garantizar el principio de la preferencia comunitaria, y un 
sistema de medidas de incentivación para facilitar la salida al exterior de los posibles excedentes que se pudieran originar-las llamadas restituciones a la exportación.

En tercer lugar, una política de estructuras, destinada a los agricultores para ayudarles a mejorar la estructura de sus explotaciones -programas de mejoray elevar sus niveles de formación profesional, así como para facilitar la incorporación de los jóvenes a la actividad agraria. Esta política tenía también un componente territorial en lo relativo a las medidas destinadas a las zonas de agricultura de montaña para compensar a los agricultores ubicados en ellas.

La financiación de esos instrumentos ha venido correspondiendo desde entonces a un fondo comunitario -el FEOGA (Fondo Europeo de Orientación y Garantía Agrícolas)- dividido en dos secciones: una, para la política de precios y mercados (sección Garantía) y otra, para la política de estructuras (sección Orientación).

La definición y formulación de la PAC le ha correspondido a las instituciones comunitarias, principalmente al Consejo de Ministros de Agricultura y a la Comisión, que han sido los encargados de aprobar los reglamentos y directivas necesarios para llevar a cabo la regulación del sector agrario de la entonces CEE y ahora de la UE. En ese proceso han intervenido dos tipos de organismos consultivos: los ya mencionados «comités consultivos agrícolas» y los llamados «comités de gestión». Los primeros, uno por cada OCM, están formados por representantes de los agricultores -siendo designados por el COPA/COGECA, el órgano máximo de representación sindical y cooperativa reconocido oficialmente por la Comisión Europea para realizar tareas de concertación (4)- y por representantes de otros grupos de intereses, como los consumidores y las industrias agroalimentarias -a través de sus correspondientes organizaciones representativas. Aunque los informes de estos «comités agrícolas» no tienen carácter vinculante, la Comisión ha estado siempre interesada en contar con una posición favorable del correspondiente comité a la hora de consultarle sobre

(4) El COPA (Comité de Organizaciones Profesionales Agrarias) está formado por más de treinta organizaciones profesionales agrarias de ámbito nacional y orientación general, actuando como el representante oficial del sindicalismo agrario europeo. El COGECA (Comité General del Cooperativismo Agrario) agrupa a una veintena de federaciones nacionales de cooperativas agrarias de los distintos países miembros de la UE. Ambos comités se coordinan para designar a los representantes que les corresponden en los comités consultivos agrarios. 
algún proyecto de reglamento antes de que éste pase al Consejo de Ministros, dado que dichos comités son fuente de legitimidad social de la PAC ante los agricultores.

Por el contrario, los informes de los «comités de gestión», que están formados por técnicos designados por los ministerios de Agricultura de cada país miembro, tienen influencia directa en el proceso de formulación de la PAC, ya que si un proyecto de reglamento no obtiene el informe favorable del correspondiente comité, puede obligar a la Comisión a tener que elaborar otro nuevo. La creación de estos «comités de gestión»-cuya constitución no estaba prevista en el Tratado de Roma- ha respondido al deseo del Consejo de Ministros de ejercer un control indirecto sobre la Comisión en la elaboración de los reglamentos y directivas, reduciendo así el amplio margen de autonomía y el protagonismo que había adquirido el órgano ejecutivo de la UE (5).

Una vez aprobados y publicados en el Diario Oficial de la CEE, los reglamentos y directivas son aplicados en los distintos Estados miembros. Para el caso de los reglamentos relativos a la gestión de los distintos mercados - muchos de ellos aprobados directamente por la Comisión Europea por delegación expresa del Consejo de Ministros-, su aplicación es inmediata, no siendo necesaria la aprobación de una norma nacional. Sin embargo, las directivas necesitan de una norma nacional de desarrollo para que puedan ser aplicadas, ocurriendo lo mismo con los reglamentos relativos a la política de estructuras -reglamentos marco-, ya que ambos dejan un margen amplio para que su contenido sea desarrollado por los gobiernos nacionales. El protagonismo de estos gobiernos ha ido en aumento conforme comenzó a introducirse en la gestión de la PAC el principio de cofinanciación de algunos programas, tal como veremos más adelante.

(5) No hay que olvidar, que la Comisión tiene entre sus principales funciones la de iniciativa legislativa, de modo que todo proyecto de reglamento o directiva tiene que partir de ella, debiendo el Consejo de Ministros conformarse con debatir los documentos elaborados por la Comisión. Asimismo, hay que tener en cuenta que, a medida que se fue desarrollando el sistema de regulación de los mercados a través de las OCM, los numerosos reglamentos de gestión coyuntural serían directamente aprobados por la Comisión por delegación expresa del Consejo. Con la creación de los «comités de gestión», el Consejo de Ministros recuperaba parte de su poder estableciendo un control sobre los trabajos de la Comisión. 


\section{El contexto de crisis de la P.A.C.}

Entre los años sesenta y setenta la agricultura europea experimenta un incremento de la producción agraria sin precedentes, de modo que, a comienzos de los ochenta, la Comisión Europea reconoce en un informe titulado «Orientaciones para la agricultura europea» (1981) la existencia de problemas serios de excedentes agrícolas en algunos sectores, como los cereales, leche y carne de vacuno, problemas que estaban haciendo inviable la financiación de la PAC con cargo al presupuesto comunitario. Esos tres sectores absorbían por sí solos el $59 \%$ del presupuesto del FEOGA.

En ese informe, propiciado por el nuevo contexto de una CEE ampliada a nueve-entre los cuales la incorporación del Reino Unido significaba la presencia de un influyente discurso de control del gasto y de revisión de los mecanismos hasta ese momento vigentes en la definición de la PAC-, la Comisión reconocía, además, que la PAC había producido problemas de desigualdad entre las regiones europeas y entre los diferentes grupos de agricultores, admitiendo el escaso desarrollo de la política estructural. Por todo ello, el informe planteaba la necesidad de introducir mecanismos de corrección en la PAC para hacerla económicamente viable y adecuarla al estado real de los mercados mundiales y a los cambios que se estaban produciendo en la demanda interna.

El verdadero problema no era sólo el del coste elevado de la PAC, sino que la lógica implícita en la política de precios y mercados era una lógica infernal para el presupuesto comunitario, imposible de frenar sin cambiar los mecanismos de funcionamiento de dicha política. En efecto, la política de precios de garantía, que se había mostrado como un mecanismo eficiente para incentivar la producción agraria incluso en zonas con características agroclimáticas poco adecuadas, se convertía, una vez alcanzada la autosuficiencia alimentaria, en un mecanismo que, al actuar al margen de la lógica del mercado, era un eficaz generador de excedentes. Por su parte, el sistema de restituciones a la exportación -mecanismo utilizado para dar salida al mercado mundial a tales excedentes- se convertía en una carga excesiva para el presupuesto comunitario una vez que los excedentes agrícolas comenzaron a adquirir un volumen desorbitado en algunos sectores, además de los efectos distorsionadores que dicho sistema tenía sobre los mercados mundiales al empujar hacia abajo los precios de los productos de tales sectores excedentarios. 


\section{Las primeras medidas correctoras de la PAC}

A principios de los años ochenta, el Consejo de Ministros de Agricultura de la CEE decide ya adoptar algunas medidas correctoras en lo que podríamos considerar una primera reforma de la PAC, si bien todavía una reforma que no alteraba en profundidad sus principios básicos, pero que flexibilizaba la aplicación de algunos de ellos. Así, el principio de solidaridad financiera es flexibilizado al crearse tasas de corresponsabilidad en el sector lácteo y en el de cereales, con la finalidad de que los agricultores participen en la financiación de los excedentes originados en dichos sectores, fijándose también cantidades máximas garantizadas -denominadas «umbrales de garantía»- en el sector de oleaginosas, por encima de las cuales los precios de garantía se debían reducir gradualmente. Se introduce en el vino la destilación obligatoria (conversión de vino en alcohol) de ciertas cantidades fijadas en cada campaña, sin dar lugar a derecho alguno de garantía. La introducción de cuotas de producción en el sector lácteo, asignadas a cada Estado miembro para que éste las redistribuyera entre sus ganaderos, fijándose importantes sanciones económicas para el caso de superarlas, también era una forma de flexibilizar los principios básicos de la PAC. Finalmente, se aprueban medidas destinadas a desincentivar a los agricultores en la utilización de los organismos públicos de intervención, como, por ejemplo, aplazar el pago de la producción vendida a dichos organismos, endurecer las normas de calidad exigidas o acortar los plazos de entrega, todo ello para hacer que el agricultor opte por vender su producción en el mercado antes que a los organismos de intervención.

En 1985, la Comisión Europea presenta el informe sobre «Perspectivas de la PAC», denominado Libro Verde, en el que se define el marco de la evolución de la agricultura europea en el horizonte del año 2000 y se plantea la necesidad de darle un giro importante a la PAC, un giro que fuera más allá que la simple adopción de medidas correctoras, tal como había ocurrido hasta ese momento. La preocupación manifestada por la Comisión en dicho informe hay que enmarcarla en un contexto caracterizado, de una parte, por la inminente ampliación de la CEE a países mediterráneos de gran potencial agrícola, como España -que se adhirió en enero de 1986-, cuya presencia hacía inviable económicamente la aplicación de una PAC sin cambios. De otra parte, la entrada en vigor del Acta Unica en 1987 -que significaba la adopción de nuevas políticas 
comunes además de la PAC-, hacía que las autoridades comunitarias plantearan la necesidad de priorizar el gasto, proponiendo recortar el presupuesto de la PAC y destinar fondos al desarrollo de esas otras políticas -como el programa marco de investigación, los programas de intercambio en el terreno de la educación universitaria o los programas de desarrollo regional financiados con cargo al fondo estructural FEDER.

El mencionado informe propone, concretamente, establecer un eficaz control de la producción agraria a través de una reducción de los precios de garantía y la extensión al resto de las OCM de las tasas de corresponsabilidad hasta entonces reducidas a sólo dos sectores, así como introducir algún mecanismo que discipline el crecimiento del gasto agrícola para hacerlo controlable. Asimismo, el citado informe señala por primera vez la conveniencia de aplicar políticas que complementen las de precios y mercados a fin de diversificar las rentas de los agricultores.

Paralelamente al mencionado Libro Verde sobre la PAC, la Comisión Europea elabora un informe sobre «El futuro del mundo rural» (1988), que, además de ser un contrapunto a los debates más coyunturales que se estaban desarrollando en torno al gasto agrícola, se convertirá rápidamente en el documento básico de referencia para una nueva forma de pensar los problemas de la agricultura y el mundo rural, al sintonizar con un nuevo estado de opinión que estaba emergiendo con fuerza en la sociedad europea de los ochenta. En efecto, temas como el de la necesidad de fijar población en el mundo rural como garantía para mantener el modelo de asentamiento y el paisaje característicos de la sociedad europea, así como el de la conservación y protección del medio ambiente, significaban un cambio importante en el terreno del discurso dentro de la Comisión Europea, pasándose de un discurso claramente productivista, como era el que había dominado en los años sesenta y setenta, a otro en el que se plantean los problemas del mundo rural de forma más amplia e interdependiente. La importancia de ese documento de reflexión radica en su impacto sobre las comunidades científicas, académicas, sindicales y políticas de la Comunidad Europea, iniciándose un giro fundamental a la hora de debatir sobre los problemas de la agricultura y el mundo rural. Asimismo, dicho documento sirvió también para que la propia Comisión Europea adoptara algunas iniciativas, como el programa LEADER, para fomentar experiencias de desarrollo rural en distintas regiones del territorio europeo. 
Respecto al tema de la reforma de la PAC, la Comisión, sobre la base del mencionado Libro Verde, elaboró un paquete de medidas de reforma -el llamado «paquete Delors», por aprobarse bajo el mandato del francés Jacques Delors como presidente de la Comisión-, de cuya importancia es buena prueba el hecho de que tuviera que ser aprobado por el Consejo de jefes de Estado y de Gobierno en 1988 ante la imposibilidad de llegar a un acuerdo en el seno del Consejo de Ministros de Agricultura. Entre esas medidas, algunas de corte similar a las de la reforma anterior, pueden destacarse las siguientes: a) una reducción de los precios de garantía en un 6\%; b) la aplicación de estabilizadores de la producción, lo que significaba en la práctica la reducción indirecta de los precios de garantía cuando en una OCM se sobrepasara una determinada cantidad máxima; c) la concesión de primas para el arranque de viñedo; d) la prórroga del sistema de cuotas de producción en el sector lácteo; e) la aplicación de un programa de retirada de tierras de la producción (set aside), así como otro de extensificación y de reconversión para sustituir cultivos excedentarios por otros que no lo fueran; f) la fijación de un límite máximo a los gastos globales ocasionados por la política agraria común, introduciéndose así un criterio de disciplina en la elaboración y posterior aplicación del presupuesto de la PAC, y g) la reforma de los fondos estructurales (FEDER, FSE y FEOGA/orientación), dividiendo el territorio de la CE en zonas por objetivos - cinco tipos de zonas-, según las distintas causas de sus problemas y hándicaps estructurales.

A pesar de estas medidas, la Comisión Europea vuelve a elaborar en 1991 un nuevo documento - «Evolución y futuro de la PAC», conocido como Primer Informe McSharry, que era el nombre del comisario de Agricultura de la $\mathrm{CE}-$, en el que se reconoce el fracaso de la primera reforma y el incremento vertiginoso de un gasto agrícola imposible de detener. Como muestra baste señalar que en 1991 se había incrementado en un $20 \%$ respecto al año anterior, y en 1992 se tenía que incrementar en un 12,5\% para hacer frente a los compromisos de la PAC.

En ese documento se introducen los principios del nuevo discurso sobre la agricultura y el mundo rural que habían sido expuestos en el anterior informe sobre «El futuro del mundo rural» ya mencionado, vinculándolos ahora a la reforma de la PAC. La plurifuncionalidad de la agricultura y de los agricultores -no sólo en sus aspectos de producción de alimentos, sino como actores fundamentales en la protección y conservación del medio ambiente-, el mante- 
nimiento de población agrícola en el medio rural como base de vertebración y cohesión sociales y de articulación del tejido productivo, la diversificación de las actividades económicas para complementar las rentas de los agricultores y como fuente de desarrollo de las zonas rurales, la introducción de criterios medioambientales en la política agraria o la necesidad de abordar programas de reforestación para devolver a las tierras agrícolas su antigua vocación forestal, son algunas de las ideas que aparecían en dicho documento y que expresaban la nueva filosofía. A ello se añadía una propuesta que constituía un cambio radical en los mecanismos de protección a los agricultores, cual era sustituir los precios de garantía por ayudas directas, moduladas en cuantía según el tamaño de las explotaciones y con limitaciones en sus niveles máximos.

\section{La reforma de la P.A.C.}

En 1992 se aprueba la reforma de la PAC, introduciendo importantes cambios en los mecanismos de protección y regulación de algunas de las más importantes OCMs, cambios que suponen un giro sustancial en lo que se venía aplicando hasta ese momento. Antes de pasar a explicar el contenido de dicha reforma, dedicaré unas líneas a comentar algunos elementos del contexto social y político en que se formula.

\section{El contexto de la reforma}

Hay que señalar, en primer lugar, la convicción en distintos foros comunitarios -especialmente en el ámbito de la Comisión- de que era necesario dar un giro fundamental a la PAC, dado que los mecanismos correctores no habían dado el resultado previsto para frenar el gasto agrícola.

En segundo lugar -y como lo prueban documentos elaborados por la Comisión del tipo de los ya comentados sobre «El futuro del mundo rural»que ese convencimiento iba más allá de una simple preocupación por los problemas coyunturales de reducción del gasto, extendiéndose a la elaboración de un nuevo discurso sobre la agricultura y el mundo rural que permitiera darle a la PAC una nueva legitimidad social entre la población europea. 
En tercer lugar, que era cada vez mayor la demanda de la opinión pública europea -manifestada a través de las organizaciones de consumidores y las asociaciones ecologistas, así como de influyentes asociaciones del comercio y la industria- para que se introdujeran nuevos criterios de regulación del sector agrario a fin de adaptarlo mejor a la realidad de los mercados mundiales, primando la calidad de la producción, el equilibrio territorial y la protección del medio ambiente, una vez comprobados los efectos negativos del modelo de intensificación productiva.

En cuarto lugar, que la previsible ampliación de la UE a los países del Este europeo constituía un elemento importante para apoyar la necesidad del cambio, ya que haría inviables económicamente los actuales mecanismos de protección de la PAC, aumentando así la conciencia de los distintos Estados miembros sobre la necesidad de la reforma.

Por último, que la presencia de importantes divisiones en el seno de la representación profesional de los agricultores - de un lado, el COPA cada vez tenía más dificultades para llegar a definir una posición común entre la diversidad de los intereses allí representados, y de otro, la CPE (Comisión Campesina Europea) emergía en la periferia de la representación como un nuevo actor cuyo discurso sintonizaba con los nuevos planteamientos de la Comisión respecto al mundo rural-era buena prueba de que, incluso desde los propios intereses agrarios, la necesidad de reforma era algo que estaba presente en ellos, aunque las distintas organizaciones sindicales pudieran discrepar de las orientaciones a seguir (6).

Todo ese contexto favorable a la reforma de la PAC se vería acelerado por la necesidad de llegar a un rápido acuerdo entre la UE y los Estados Unidos de América en el seno de las negociaciones del GATT, negociaciones que estaban alargándose en demasía después de varios años de interminables reuniones entre los países participantes. El citado acuerdo conducía a una modificación del

(6) La CPE estaba formada por organizaciones representativas de la agricultura familiar no integradas en el COPA, manteniendo desde mediados de los años ochenta importantes discrepancias con las orientaciones de la PAC y planteando la necesidad de su reforma en profundidad. Incluso desde dentro del propio COPA algunas organizaciones miembros expresaban posiciones similares a las de la CPE. Por su parte, algunas asociaciones sectoriales por productos también se manifestaban a favor de una reforma de la PAC. 
sistema de precios de garantía de la PAC para impedir sus efectos distorsionadores sobre los mercados mundiales, modificación que, recordémoslo, ya estaba planteada en el mencionado Informe del comisario de Agricultura McSharry.

El temor a que las movilizaciones agrarias -promovidas por algunas organizaciones sindicales contrarias a que la reforma se orientara hacia criterios de modulación diferenciada en favor de la agricultura familiar- se extendieran y crearan un ambiente de inestabilidad social en un momento en que se estaban desarrollando los referéndums para la ratificación del Tratado de Maastricht (7), es un factor importante para comprender la orientación final que adoptó la reforma de la PAC, una reforma menos ambiciosa de lo inicialmente previsto por el comisario McSharry y en la que desaparecía cualquier rasgo de la modulación diferenciada exigida por las organizaciones representativas de la agricultura familiar.

La reforma de la PAC adoptada en 1992 y aplicada para un período de cinco años, giraba en torno a dos ejes: a) la reforma de los mecanismos de regulación de algunas OCMs con importante peso específico en el conjunto de la producción final agraria europea -principalmente, cereales, oleaginosas, proteaginosas, carne de vacuno, ovino y leche y productos lácteos, y tabaco-; y b) las llamadas «medidas de acompañamiento», que significaban la potenciación de antiguas medidas de política estructural y la introducción de un programa agroambiental.

\section{La reforma de la política de precios y regulación de mercados}

Este eje es el fundamental de la actual reforma de la PAC y consiste, por un lado, en la reducción gradual de los precios de garantía hasta equipararlos a los del mercado y, por otro, la concesión de ayudas directas a los agricultores para compensarles de las pérdidas ocasionadas en sus rentas como consecuencia de tal reducción. La cuantía de las ayudas se calcula en función de la superficie de la explotación del beneficiario y de un índice referido al rendimiento medio de la zona homogénea desde el punto de vista agroclimático en que estuviera aquélla

(7) El Tratado de Maastricht, aprobado en 1993, significaba un reforma en profundidad del Tratado de Roma para dar un paso adelante en la unión política y monetaria europea, por lo que exigió en algunos países, como Francia y Dinamarca, la realización de referéndums de ratificación -en otros países bastó con una ratificación parlamentaria. 
ubicada. Las ayudas son concedidas como subvenciones públicas a fondo perdido, no exigiendo contrapartida alguna al agricultor beneficiario, salvo la de dejar una parte de su explotación sin cultivar -en torno al 15\%, si bien esta superficie se fija todos los años por la Comisión Europea (8). De este modo, y al no introducirse criterio alguno de modulación ni limites máximos a la cuantía de las ayudas -como sí se establecían en los primeros proyectos de reforma-, las ayudas benefician a los agricultores que poseen mayores superficies de tierras.

Las opiniones respecto a la reforma de la PAC, una vez transcurridos los cinco años previstos desde su puesta en marcha, varían de unos grupos a otros. Así, las organizaciones representativas de la agricultura familiar consideran injustificada la no inclusión de criterios de modulación en las ayudas, denunciando las cuantiosas fortunas que están haciendo con ellas los grandes propietarios, algunos de ellos sin haber tenido relación directa con la actividad agraria. Por su parte, las organizaciones que representan a los grandes agricultores consideran que se debe evaluar el sistema actual de ayudas por lo que realmente es, o sea, un sistema destinado a compensar en un periodo de transición a los agricultores por las pérdidas ocasionadas por la reducción de los precios de garantía, considerando lógico que, al ser precisamente los grandes agricultores los más perjudicados por dicha reducción de precios, sean también ellos los que se beneficien en mayor medida con el sistema compensatorio de ayudas directas. Para estas organizaciones de tipo empresarial, introducir criterios de modulación social en políticas de mercados sería algo contradictorio con la lógica interna de este tipo de políticas. Consideran, además, que si los agricultores titulares de grandes explotaciones son los que más se benefician de los sistemas de ayudas directas, deben ser los sistemas progresivos de imposición fiscal los encargados de hacer que paguen más impuestos redistribuyendo así las rentas. No obstante, estas organizaciones empresariales expresan su preocupación por lo que entienden que es una pérdida de legitimidad social de los agricultores en tanto que empresarios, ya que reciben subvenciones sin asumir riesgo alguno.

Puede decirse que hay consenso sobre la necesidad de introducir medidas de corrección en el actual sistema de ayudas una vez finalizado en 1997 el periodo inicial de cinco años previsto. Entre las medidas correctoras

(8) De este requisito están exentos los agricultores de pequeñas explotaciones. 
parecen abrirse paso - a la vista de algunos informes elaborados en forma de proyectos de reglamento por la Comisión en el marco de la Agenda 2000la de introducir criterios de limitación máxima a la cuantía de las ayudas directas, así como la de establecer una especie de cuadro de compromisos por el que el agricultor beneficiario se comprometa a desarrollar determinadas prácticas agrícolas en su explotación.

Respecto a las otras OCMs reformadas -vacuno de carne, ovino y lechelos mecanismos de la reforma responden a criterios similares a los expuestos para las OCMs de cereales y oleaginosas, es decir, la concesión de ayudas directas -sólo que en estos otros casos se calculan en función del número de cabezas de ganado o de la cantidad de leche producida. Quedan otras OCMs sin reformar, dado que al no ser excedentarias no planteaban problemas en el ámbito de las negociaciones del GATT, algunas de ellas de gran importancia para la agricultura mediterránea -como aceite de oliva, vino y frutas y hortalizas-, y cuyos reglamentos de reforma están siendo sometidos a debate y discusión por las instituciones comunitarias en el momento de escribir este artículo.

\section{b) Las medidas de acompañamiento}

La denominación de «medidas de acompañamiento» indica ya que el paquete de medidas adjunto a la reforma de la política de precios y mercados tenía, en sus orígenes, un carácter complementario a ésta, siendo su objetivo ofrecer-con cargo al propio presupuesto de la PAC y con participación de los gobiernos nacionales-oportunidades a los agricultores para complementar sus rentas con actividades a realizar en sus explotaciones, pero no relacionadas directamente con la producción agraria.

Tres son estos programas de acompañamiento. En primer lugar, un programa de reforestación de tierras agrícolas, consistente en la concesión de ayudas a los agricultores que se comprometan a sustituir total o parcialmente la orientación productiva de sus explotaciones por una orientación forestal, devolviendo así a algunas zonas del territorio la antigua vocación forestal -de ahí la utilización del término «reforestación»- que el proceso de modernización agrícola habría desviado hacia la búsqueda de la rentabilidad inmediata vía intensificación agrícola o ganadera. Este programa concede ayudas por la 
implantación de determinadas variedades forestales y para sufragar el mantenimiento por un espacio de 25 años.

En segundo lugar, un programa de jubilación anticipada destinado a los agricultores de más de 55 años que deseen abandonar la actividad agraria y transmitir su explotación o bien a jóvenes que quieran instalarse por primera vez en la agricultura o a otros agricultores que tengan intención de ampliar la superficie de sus explotaciones. Este programa pretende contribuir al rejuvenecimiento de la población agrícola y a la creación de explotaciones agrarias con estructuras más eficientes, fijando al mismo tiempo en el medio rural una población de prejubilados con una renta suficiente para permitirles esperar a la edad de la jubilación oficial-en torno a los 65 años-y acceder al sistema general de pensiones. Este programa es extensivo a las ayudas familiares y asalariados agrícolas de más de 55 años que trabajen de forma permanente en la explotación del titular que se acoja al programa.

En tercer lugar, un programa de medidas agroambientales destinado a los agricultores que deseen introducir en sus explotaciones unas prácticas más respetuosas con el medio ambiente, que las convencionales. A diferencia de las otras medidas de acompañamiento, que estaban incluidas en algunos programas socioestructurales previos a la reforma de 1992, aunque poco desarrolladas, el programa agroambiental constituye una novedad por cuanto que por primera vez se ha planteado en el seno de la UE un conjunto articulado de medidas de carácter obligatorio para los países miembros, tendentes a la introducción de prácticas de agricultura sostenible (9). La puesta en marcha del programa agroambiental supone el establecimiento de un sistema de contratación individualizada entre el agricultor que desee acogerse al mismo y los poderes públicos -bien nacionales o regionales, según la estructura políticoadministrativa de cada país-, por el cual aquél acepta unos compromisos respecto al tipo de prácticas a realizar en su explotación a cambio de recibir una ayuda económica para compensar la menor rentabilidad que le pudiera ocasionar la opción por una agricultura sostenible.

Las medidas que componen el programa agroambiental son las siguientes.

a) Ayudas para reducir el uso de prácticas agrícolas y ganaderas perjudiciales

(9) Bien es cierto que algunas de las medidas agroambientales, como las relativas a zonas sensibles o las de extensificación, estaban incluidas en el R(CEE) 797/85 y en el 2328/91, pero lo estaban con carácter facultativo y no obligatorio como ahora. 
para el medio ambiente, como la utilización de productos agroquímicos (fertilizantes, pesticidas, herbicidas,...), la existencia de una excesiva carga ganadera o el uso de prácticas de laboreo intensivo.

b) Ayudas para fomentar una gestión de las tierras agrícolas y ganaderas más positiva para el mantenimiento de la biodiversidad tanto animal, como vegetal, y para una mejor conservación del paisaje. Entre las prácticas incluidas en este régimen de ayudas se citan expresamente las que permiten laintroducción de razas ganaderas en peligro de extinción debido a la especialización de la ganadería convencional, o las que posibilitan el retorno a prácticas tradicionales de agricultura y a la extensificación, ayudando así a evitar la desaparición de la fauna y flora autóctonas.

c) Ayudas para una adecuada conservación medioambiental de las tierras retiradas obligatoriamente de la producción en el programa de reforma de las OCMs de cereales y oleaginosas ya mencionado.

d) Ayudas para la retirada voluntaria de tierras de la producción por un periodo de larga duración -25 años-, con el compromiso de realizar prácticas de conservación medioambiental.

e) Ayudas para la realización de cursos de formación agroambiental destinados a los agricultores a través de sus organizaciones profesionales y cooperativas.

f) Ayudas para el fomento de la agricultura ecológica, destinadas no sólo a los agricultores que deseen reconvertir sus explotaciones a este tipo de agricultura alternativa, sino también a jóvenes que se instalan por primera vez y optan por este modelo de explotación.

A diferencia de la reforma del sistema de precios y mercados y la introducción del sistema de ayudas directas, que, como se indicó, es financiado en su totalidad por el presupuesto comunitario con cargo a la sección Garantía del FEOGA, la medidas de acompañamiento son cofinanciadas entre la UE y los Estados miembros, en unos porcentajes variables según las zonas (10), lo que ha significado un elemento de complejidad importante a la hora de su aplicación.

Así como existen distintas valoraciones sobre el impacto y resultados de la

(10) En las zonas de objetivo n.1, la UE contribuye con un $75 \%$ de la ayuda y el gobierno nacional con un 25\%, mientras que en el resto de las zonas la cofinanciación es del $50 \%$ cada parte. 
reforma de la PAC en lo que se refiere a la política de precios y mercados una vez transcurridos cinco años de su aplicación, no es posible, por el contrario, establecer una valoración seria y rigurosa sobre el resultado de las medidas de acompañamiento y, en particular, sobre el programa agroambiental, dado el corto espacio de tiempo que llevan aplicándose. La lentitud con la que se está produciendo su puesta en marcha en cada Estado miembro hace que no se disponga todavía de un horizonte temporal lo suficiente largo para valorar la mayoría de los programas tanto en lo que se refiere al número de beneficiarios en los distintos regímenes de ayudas, como en lo que respecta al cumplimiento de los compromisos adquiridos por el agricultor en el correspondiente contrato. En muchos países, hay programas que ni siquiera se han iniciado, y en otros apenas llevan uno o dos años de funcionamiento (11).

Esto es debido a que la aplicación de los reglamentos que componen las medidas de acompañamiento deja un amplio margen de maniobra a los Estados miembros para que fijen las prácticas que consideren sostenibles desde el punto de vista de las condiciones estructurales de las agriculturas y los ecosistemas nacionales, exigiendo, por tanto, la elaboración de una o varias normas nacionales, que, en muchas ocasiones, exige una negociación interna laboriosa entre el ministerio de Agricultura, las organizaciones agrarias y las asociaciones ecologistas. Estas normas deben ser trasladadas por los gobiernos nacionales a la Comisión Europea para recibir su homologación, lo que abre otra ronda de negociaciones a nivel europeo entre ésta y cada Estado miembro (12). Asimismo, la estructura federal de algunos Estados de la UE -como España, Italia o Alemania- hace que la elaboración de esas normas nacionales de aplicación tengan que ser también negociada con los gobiernos regionales, debiéndose incluso de elaborar otras normas adicionales para establecer los procedimientos administrativos necesarios para la concesión de las correspondientes ayudas. El hecho de que la parte de financiación que corresponde al Estado miembro -el 50 o el $25 \%$ según las zonas- sea, en el caso de estructuras federales,

(11) El programa de reforestación era el más avanzado de los tres, pero la coincidencia de tres años terribles (1992-1995) de sequía en los países mediterráneos -que eran en los que más interés podía tener dicho programa, dado el problema de erosión de los suelosha sido un factor limitante.

(12) La negociación suele hacerse en el seno del llamado comité STAR, que es un comité de gestión para los temas agroambientales. 
cofinanciada a su vez por el gobierno regional, introduce otro elemento de complejidad en la negociación previa a la definitiva puesta en marcha del programa.

\section{A modo de conclusión: nuevas políticas y nuevos actores sociales}

La actual reforma de la PAC es un proceso abierto que no ha hecho más que comenzar. En ese proceso se irá avanzando en la introducción de nuevos mecanismos de regulación del sector agrario para adaptarlo a la realidad de unos mercados internacionales cada vez más abiertos y para que responda a las nuevas demandas de la sociedad europea. Dentro de esas nuevas demandas, la aspiración de contar con unos espacios rurales vivos y dinámicos, así como con un medio ambiente de calidad, explica la emergencia de nuevas políticas complementarias a las tradicionales de estructuras y de regulación de los mercados. Este planteamiento ha sido recogido en el Documento Agenda 2.000 elaborado por la Comisión Europea y que se encuentra ahora en fase de discusión por los Estados miembros.

Entre las nuevas políticas, la agroambiental a que se ha hecho referencia en este trabajo constituye un primer intento de definir a nivel europeo una política que regule las relaciones entre agricultura y medio ambiente para posibilitar la continuidad de los actuales sistemas de producción agraria evitando los efectos negativos de tales sistemas sobre el entorno natural.

Las políticas de desarrollo rural, destinadas a favorecer la diversificación de actividades en el medio rural, movilizando todos los recursos disponibles -agrarios y no agrarios- con el objetivo de mantener un tejido social activo en las comunidades rurales europeas, constituyen otras nuevas políticas que sobrepasan el ámbito de la política agraria. Estas nuevas políticas no han sido analizadas en este artículo -salvo la cita que se ha hecho a los programas LEADER y PRODER-dado que no forman parte de la PAC, siendo actualmente formuladas o bien como iniciativas con carácter experimental de la propia Comisión Europea o bien como programas operativos de los Estados miembros dentro de planes de desarrollo regional. En consecuencia, no puede hablarse todavía de una política europea de desarrollo rural, sino sólo de sus comienzos. La conferencia celebrada en la ciudad irlandesa de Cork en 1996 auspiciada por el propio comisario de Agricultura de la Unión Europea, fue el primer intento de 
definir los principios de una futura política europea de desarrollo rural, aún en estado embrionario. No obstante, el debate sobre si la política de desarrollo rural debe ser formulada en el marco de las políticas agrarias o ser integradas en las de desarrollo regional, es un debate abierto -y que la Agenda 2.000 no hace más que reabrir- de cuya resolución dependerá el futuro de dicha política.

En cualquier caso, estas nuevas políticas -tanto la de desarrollo rural, como la agroambiental- conducen a la redefinición del papel de la agricultura y el mundo rural en el escenario de la Unión Europea, teniendo como consecuencia un cambio en la composición de los actores sociales. Las organizaciones profesionales agrarias, hegemónicas como representantes de los agricultores y del mundo rural en general durante el periodo de la modernización productiva bajo la égide de la PAC, ven ahora reducido su protagonismo y perdido el monopolio de la representación de que habían gozado.

Nuevos actores sociales emergen en el panorama asociativo. De un lado, las organizaciones agrarias - por ejemplo, las integradas en la ya mencionada CPEcuyo discurso no productivista no sintonizaba con la política agraria dominante, se erigen ahora en interlocutores válidos para las nuevas orientaciones de la PAC. De otro, las organizaciones ecologistas aspiran a intervenir en el proceso de formulación y aplicación de la política agroambiental al mismo nivel que las organizaciones agrarias, ya que consideran que la protección del medio ambiente es algo que afecta al conjunto de la población y no sólo a los agricultores. Finalmente, nuevos actores no agrarios emergen en el ámbito del desarrollo rural, participando en las distintas estrategias de desarrollo económico y compartiendo con los agricultores la responsabilidad del futuro de los espacios rurales.

La emergencia de nuevos actores sociales refleja la existencia de una sociedad plural y diversificada como es la sociedad rural europea de hoy, una sociedad en la que los agricultores ya no son el grupo social dominante, sino una minoría entre otras, un grupo social como otros que debe compartir en posición de igualdad los problemas del mundo rural y la búsqueda de soluciones. La participación de los agricultores en las políticas agroambientales y en las iniciativas de desarrollo rural tal vez sea uno de los retos que tienen planteadas muchas zonas rurales europeas en las que la población agrícola todavía es importante; de su integración en esas políticas depende quizá que los agricultores salgan del gueto en que los discursos agraristas le habían metido bajo la seducción de que formaban parte de un mundo singular y, por ello, merecedor de un 
tratamiento privilegiado. La apertura de los mercados agrícolas y la reforma de los mecanismos de protección colocan a los agricultores en una posición real y no ficticia, una posición como la de cualquier otro grupo socioprofesional que ha de buscar -sin abandonar el objetivo de mejorar la competitividad de sus explotaciones- fuentes complementarias de rentas y apoyar la creación de nuevas riquezas en el mundo rural a través de la diversificación de las actividades productivas. 